\title{
Social Needs and Happiness: A Life Course Perspective
}

\author{
Vera L. Buijs ${ }^{1,2}(\mathbb{D}) \cdot$ Bertus F. Jeronimus ${ }^{2,3}(\mathbb{D}) \cdot$ Gerine M. A. Lodder ${ }^{1,4}(\mathbb{D}$. \\ Nardi Steverink ${ }^{1,5}$ (D) . Peter de Jonge $\mathrm{e}^{2,3}$ (D)
}

Published online: 10 July 2020

(C) The Author(s) 2020

\begin{abstract}
Both the fulfilment of affection, status, and behavioral confirmation needs and their role in happiness may differ along the adult lifespan. We examined age-graded differences in (a) the fulfilment of the need for affection, status, and behavioral confirmation, (b) disharmonious profiles of need fulfillment (e.g., high affection but low status), and (c) the associations between these needs and happiness. Data from 11,406 Dutch respondents (age range 18-87 $(M=44.82, S D=14.62), 67 \%$ female $)$ were collected via hoegekis.nl and categorized over six age groups (early, young, middle-aged and late adults, young-old and oldest-old). Agegraded differences in social need fulfilment and their link to happiness were examined using regression analyses. Need fulfillment profiles were identified with LCA cluster analyses. Age-graded differences in social need fulfilment were virtually absent (Cohen's $d=0.20$ or smaller) and their link with happiness was stable across the age groups. Social need fulfilment profiles were harmonious as people reported either low, middle, or high need fulfilment in general, irrespective of age. The idea that different social needs are more important in different phases of adult life received only weak support in our data. No strategic investment in specific social needs was observed (no substitution-effects).People typically differed in their capacities to fulfil their affection, status, and behavioral confirmation needs in general, regardless of age. The implications of these results for the social production function theory of wellbeing and socioemotional selectivity theory are outlined in the discussion.
\end{abstract}

Electronic supplementary material The online version of this article (https://doi.org/10.1007/s 1090 2-020-00287-9) contains supplementary material, which is available to authorized users.

Vera L. Buijs

v.1.buijs@rug.nl

1 Department of Sociology/ICS, University of Groningen, Bouman Building B.011, Grote Rozenstraat 31, 9712 TG Groningen, The Netherlands

2 Department of Developmental Psychology, University of Groningen, Groningen, The Netherlands

3 The Interdisciplinary Center Psychopathology and Emotion regulation (ICPE), University Medical Center Groningen, University of Groningen, Groningen, The Netherlands

4 Department of Developmental Psychology, Tilburg University, Tilburg, The Netherlands

5 Department of Health Psychology, University Medical Center Groningen, University of Groningen, Groningen, The Netherlands 
Keywords Subjective well-being $\cdot$ Happiness $\cdot$ Adulthood $\cdot$ Social needs $\cdot$ Social production function theory $\cdot$ Socioemotional selectivity theory

\section{Introduction}

Social needs are universal and their fulfilment is considered to be a key human motivation and perquisite for happiness (Lindenberg 2013; Maslow 1943; Tay and Diener 2011). The negative health consequences of unfulfilled social needs (e.g., loneliness) have an effect size on par with the effects of smoking and obesity (Holt-Lunstad et al. 2015; Steptoe 2019). Individual differences in social need fulfilment have been related to personality (Ormel, et al. 2017), physical health (Steverink and Lindenberg 2006), and social resources, which are all known to change along the life course (Wrzus et al. 2013). Agegraded differences in personality and social resources typically reflect transitions in or out of social roles as partner, parent, and worker (e.g., Klärner et al. 2016; Mund et al. 2018; Rözer et al. 2017; Van den Bogaard et al. 2014). Social needs and their fulfilment may, therefore, also differ over the life course following these normative social role transitions.

Age-graded differences in social contexts across the life course reflect processes such as changes in friendship networks after finishing high school (Buote et al. 2007), which may influence social need fulfilment. Most research on social contexts and need fulfilment are primarily focused on young adults (e.g., Buote et al. 2007) or retired populations (e.g., Ten Bruggencate et al. 2017). Furthermore, previous studies often regarded social need fulfilment as indicator of happiness (e.g., Bos et al. 2016; Cramm et al. 2012). This approach neglected the role of specific social needs in happiness (e.g., Max-Neef 1992) and how their fulfilment differs across the life course (see for exceptions, Ormel et al. 2017; Steverink and Lindenberg 2006).

The present study aimed to enrich the literature by unraveling potential age-group differences in the fulfilment of the need for affection, status, and behavioral confirmation, and their connection with happiness. Below we first introduce these social needs and our underlying theoretical framework, followed by a life course perspective on age-graded differences in need fulfilment. Finally, we outline why differences in the fulfilment of these social needs might be linked to happiness.

\subsection{Social Needs}

Social needs have been conceptualized and investigated in various ways (e.g., Deci and Ryan 2008; Lindenberg 1996, 2013; Maslow 1943; Max-Neef 1992; McClelland 1985). Although there is some overlap between these theoretical approaches on social needs (e.g., Ormel et al. 1999; Steverink et al. 2019), the possibility of substitution between different social needs and a life course perspective on such processes is rather unique to the Social Production Function (SPF) theory (Steverink and Lindenberg 2006). For example, both substitution between social needs and concrete predictions on divergent life course trajectories do not directly follow from the self-determination theory (e.g., Deci and Ryan 2008; Wehmeyer et al. 2017). SPF theory is therefore particularly useful for the purposes of our study.

SPF theory assumes that humans are motivated to improve their happiness via the fulfilment of basic physical and social needs which can be ordered hierarchically (Fig. 1): subjective well-being as the superordinate level (level 1) emerging from social and physical well-being (level 2) which can be derived via the fulfilment of three social and two 


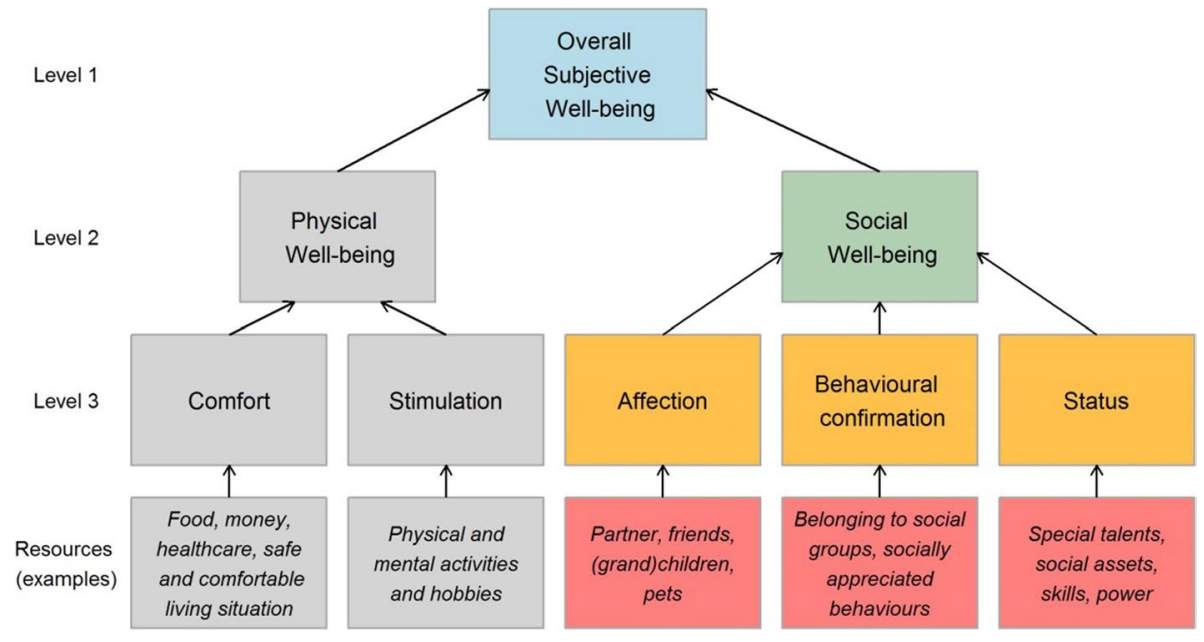

Fig. 1 Overview of need hierarchy according to SPF theory. Physical needs (in grey) are not included in this paper. The resources display examples of ways to fulfil the needs above. Note that these resources are not necessarily only useful to satisfy the need above, they could also be used to fulfill other needs. For example, a friend can attend to you (affection), but also make you feel like you belong to a social group (behavioral confirmation). Adapted from Ormel et al. (1999)

physical needs (level 3) (see Ormel et al. 1999). SPF theory assumes that these three levels of subjective well-being ${ }^{1}$ are universal thus all human beings share these needs. However, the fulfilment of the social and physical needs requires resources like money and social relations, which may differ between individuals, and vary according to time, place, and circumstances (see for comparable theories Max-Neef 1992, p. 199). Consequently, the needs themselves are universal, but the resources and restrictions people have and encounter to fulfil their needs may differ, as illustrated in Fig. 1.

Although SPF theory is about both social and physical needs, the present study has been specifically focused on the three social needs of SPF theory (see also Steverink and Lindenberg 2006; Steverink et al. 2019). Although the fulfilment of physical needs may also be expected to differ over the life course, these physical needs are less dependent on the social context and, therefore, considered to fall outside the scope of the present study. First, affection is the sense that people care about you, regardless of your status and behavior. The need for affection can be fulfilled by relationships that provide, among others, love, trust, and understanding (cf. Maslow 1943). This need is comparable to the need for relatedness (Deci and Ryan 2008), need for intimacy (McClelland 1985), and need to belong (attachment; Baumeister and Leary 1995). Second, behavioral confirmation is the sense that you do the right things according to the people in your social environment. Relationships that provide approval, appreciation and encouragement fulfill the need for behavioral confirmation, which consists of, among others, doing good, being useful, contributing to a common goal, and being part of a group. The need for behavioral confirmation is comparable to the

\footnotetext{
1 Subjective well-being and happiness are often used interchangeably in scientific literatures, as both capture how one feels and thinks about one's life (Medvedev and Landhuis 2018; Diener et al. 2009). SPF theory uses subjective well-being, but we use happiness throughout this paper because that is how we operationalized our measurement.
} 
need to belong (feeling accepted by the group; Anant 1967). Third, status is the sense that you achieve more than others, and are known for your achievements, skills or assets. Status needs can be fulfilled by relationships that provide respect, praising, and influence (cf. Maslow 1943) and compare to the need for competence (Deci and Ryan 2008) or need for achievement (McClelland 1985). Taken together, according to SPF theory, the fulfilment of social needs is dependent on the reaction from others on who a person is (affection), what a person does (behavioral confirmation) and what a person has (status) (see Steverink and Lindenberg 2006).

Importantly, SPF theory further states that the resources used to fulfil these social needs can be substituted. For instance, after a love relationship breaks down, someone can shift focus to family relations to fulfil the need for affection. Moreover, SPF theory indicates that people might even be able to substitute one social need for another when the latter is easier for them to fulfil. For example, when someone loses her job and thereby loses status, this person might start to focus more on fulfilling the needs of affection and behavioral confirmation instead, in order to maintain similar levels of happiness. The increased fulfilment of these other needs might yield a relatively stable level of happiness, despite the loss of status. For example, affection was relatively more important for the happiness of people with a lack of status than for their high status peers (Nieboer and Lindenberg 2002). In line with SPF theory and substitution processes, we therefore expect that people can have different strategies and resources to fulfil social needs, which may result in different profiles of need fulfilment.

\subsection{Levels of Need Fulfilment over the Life Course}

To examine differences in social need fulfilment between age groups, we distinguish between six age groups in the Dutch population characterized by specific goals and life events (Table 1). Generally, the adult life course is divided into three main developmental periods: young adulthood, middle adulthood, and late adulthood (Sigelman and Rider 2018). In young adulthood we distinguish between early and young adults (Arnett 2000), in middle adulthood, between middle and late adults (Lachman 2004) and in late adulthood we distinguish between young and oldest-old (Wink and James 2007). Although cutoffs are always arbitrary and there is no consensus on these categorizations in the literature, the age groups we used do represent commonly distinguished developmental stages in adulthood (e.g., Ebner et al. 2006). Based on the 'typical' life course in the Netherlands, we can expect differences in need fulfilment between these six age groups. Combined with the variation in social contexts along the life-course and availability of resources such as money, friends and time for family we expect different social need fulfilment profiles, as outlined below.

\subsubsection{Affection}

The need for affection is best satisfied by close relationships including partners, family, and best friends. Emerging adults (age 18-25) start to become more independent from their parents (Arnett 2000) and typically still live in their parental home or left home recently (Statistics Netherlands 2015). Simultaneously, in the transition from secondary to tertiary education, a temporary loss in affection can be expected from changes in friendship networks (Buote et al. 2007; Oswald and Clark 2003). 


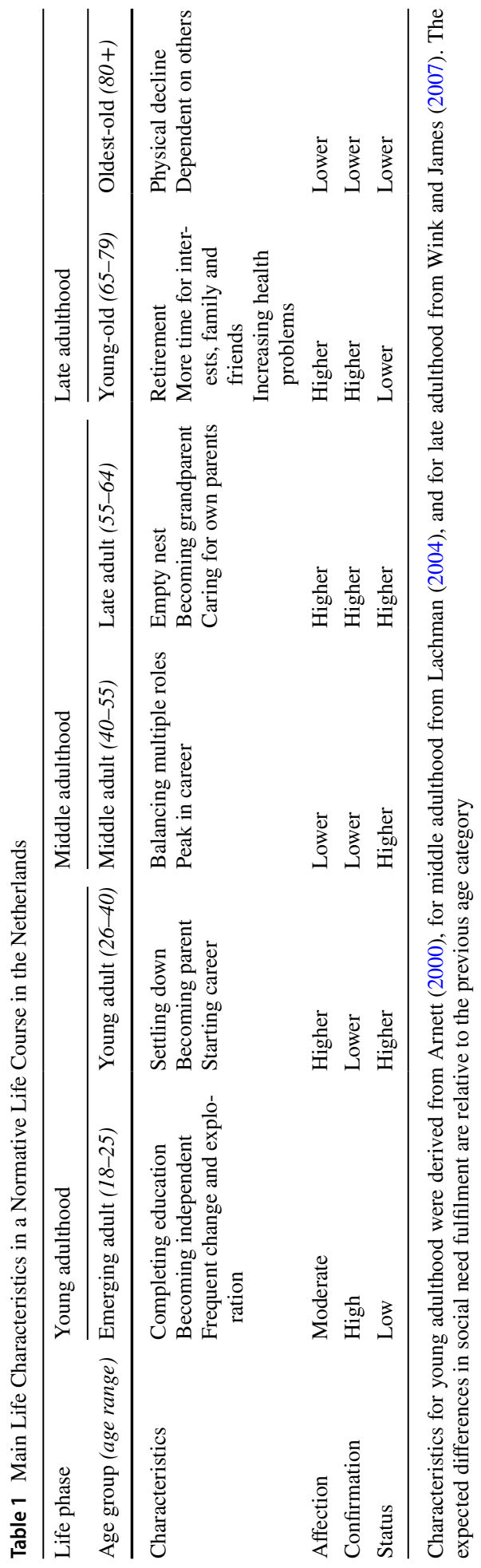


Most young adults (age 25-40), in turn, settle down, and many start families with children (Statistics Netherlands 2015), and partners and children are likely to result in higher average levels of affection. In middle-aged adulthood (age 40-55), when potential offspring grows older, we can expect less affection from them as compared to when these children were younger (Luthar and Ciciolla 2016; Marceau et al. 2015). Over 75\% of the Dutch have children by the age of 45 (Statistics Netherlands 2017a, b). Most middle-aged adults also have less leisure time to spend with family and friends, as they reach their peak in work position and earnings (Lachman 2004).

Next, in late adulthood (age 55-65), many parents experience the empty nest syndrome, as their children leave the parental home (Statistics Netherlands 2004; Lachman 2004). The empty nest could result in lower affection levels, however, it may also be compensated by the arrival of grandchildren and higher marriage satisfaction (Gorchoff et al. 2008). Providing care to elderly parents and grandchildren is likely to increase levels of affection fulfillment as well (Brody 2004).

After retirement, the young-old (age 65-80) have more time available to spend with family and friends (Lachman 2004), suggesting an increase in average fulfillment of affection needs. Finally, for the oldest-old (age 80+) affection may become difficult to obtain, as physical decline can hamper possibilities for social contact and because friends pass away. Indeed, levels of affection have been shown to decline from 80 years onwards (Steverink 2001) while loneliness sharply increases after the age of 80 (Dykstra 2009). These processes may reflect a lack of social contacts, possibly related to relationship losses due to physical health and loss of network members, and thus a lack of resources to fulfill all social needs.

\subsubsection{Behavioral Confirmation}

Second, the need for behavioral confirmation is mostly satisfied in group settings. We expect emerging adults to have relatively high levels of behavioral confirmation as they spend most of their time surrounded by others, including at school, home, work, or in sport contexts that provide ample opportunities for approval and encouragement (Arnett 2000).

In young adulthood, getting a job also shapes one's social position and typically results in a network of co-workers and new friends (Morrison 2002). Furthermore, many young adults become parents and receive behavioral confirmation via parenthood and associated social norms (e.g., Bernardi and Klärner 2014; Park 2002; Turnbull et al. 2016), while networks start to segregate between parents and non-parents (Belsky and Rovine 1984; Klärner et al. 2016). Consequently, young adults who start a family will, on average, spend less time with old friends and acquaintances, but experience new opportunities to connect with other parents instead (Belsky and Rovine 1984; Rözer et al. 2017). Moderate levels of behavioral confirmation may be expected as young adults are surrounded by others in similar situations, but contact with their old friends decreases.

Middle-aged adulthood, even more than young adulthood, is characterized by the balance of multiple social roles, like being a parent, employee and friend (Lachman 2004). Role competition might negatively affect levels of behavioral confirmation, as a child or co-parent may expect that more time is spend with the family, whereas employers and colleagues may actively discourage parents to choose their children over their work. This might already play a role in young adulthood as well, but when careers peak in middle adulthood, we expect these pressures to become stronger. Role competition may decrease the contextual support middle adults experience, which, combined with a shrinking social 
network (see Wrzus et al. 2013), may result in fewer opportunities for support and approval, thus lower fulfilment of their need for behavioral confirmation compared to young adults.

For late adults role competition will likely decrease, as career promotions are likely to become less important and children become more independent. Although the network size still decreases (Wrzus et al. 2013), opportunities for behavioral confirmation might slightly increase in this age category.

After retirement, the young-old might focus more on gaining behavioral confirmation to compensate for loss in status. Compared to peers between 54 and 69 who did not yet retire, retirees provided more tangible help, spent more time volunteering and increased their organizational memberships (Van den Bogaard et al. 2014), suggesting that they indeed tried to produce higher levels of behavioral confirmation. If age increases further, physical decline (Hall et al. 2017) makes it more difficult to gain behavioral confirmation. The oldest-old have, on average, less opportunities to participate in society and, for instance, to help others. Social participation, and hence opportunities to produce behavioral confirmation, has been shown to decrease in old age (Huxhold et al. 2013).

\subsubsection{Status}

Third, status needs are mostly fulfilled via specific skills and career positions. Emerging adults may be expected to have low status levels, as they often still depend on parental support (e.g., for money; Jones et al. 2006) and have to achieve specific skills, via training. At age 20,70\% of the Dutch still follow education (Dutch Ministry of Education, Culture and Science 2013). Average status fulfillment levels are likely to increase from young adulthood onwards, as most people start their career in their twenties (Statistics Netherlands 2019).

When in middle adulthood most people reach their peak in work position and earnings (Lachman 2004), status fulfillment levels are likely to be higher too, especially for men, as they work most hours in this age group (Statistics Netherlands 2017a, b). Work is an indicator for status, and working more hours is likely to improve status on average.

In late adulthood, most people are already over their peak in work position and salary but many still work. Additionally, late adults are supposed to have increased wisdom and practical intelligence, and a strong sense of mastery (Lachman 2004), which is likely to fulfill status needs in late adults as well. In Western societies, status is mainly related to occupational status, which means that it is much harder to achieve or maintain status after retirement (Lindenberg 1996; Steverink 2001). In a sample of people over 65 years old, status was found to be the least fulfilled social need (Steverink and Lindenberg 2006). We therefore expect that average status levels will be lower for the young-old, and even lower for the oldest-old, as they have to increasingly rely on others, and therefore become less independent.

\subsection{Need Fulfilment and Happiness over the Life Course}

We argued that levels of specific social need fulfilment can differ between age groups because of different resources or opportunities for access to certain social relations. However, the relative benefit of fulfilling a specific need (i.e., "return on investment") might also differ between age groups. For example, the fulfilment of affection needs might be less important for happiness in young and middle adulthood than for older adults. In other words, the SPF theory asserts that fulfilling social needs increases happiness similarly in 
all age groups, which can be contrasted against the perspective that the strength of the relation between levels of specific need fulfilment and experienced happiness differs between different age groups. The argument of why social need fulfillment would yield different happiness levels in different age groups based on different opportunities and resources can be found in the socioemotional selectivity theory, as outlined below.

\subsubsection{Affection and Happiness Across the Life Course}

Socioemotional selectivity theory postulates that older adults will put more energy into emotional goals because they face a limited time horizon, which makes them strive for goals that have direct emotional benefits (Carstensen 1992; Reed et al. 2014). Emotional goals include a preference for emotionally gratifying social contacts over contacts with novel social partners (Löckenhoff and Carstensen 2004, p. 1396). Interactions with acquaintances and distant friends are selectively diminished from early adulthood to old age, while people invest more in interactions with family and close friends, which in general increases the emotional closeness in the social network (Carstensen 1992). Older people are thus expected to be happier as they leverage contacts with close friends and family and we expect a stronger association between affection needs and happiness in older versus younger age groups.

\subsubsection{Behavioral Confirmation and Happiness Across the Life Course}

The relationship between behavioral confirmation and happiness may also differ over the life course. Young adulthood is characterized by identity formation and exploration (Arnett 2000; Erikson and Erikson 1998), and people derive knowledge on how to behave by comparing themselves to similar others (Suls and Wills 1991). Most young adults prioritize peer relations and friendships over other activities and adhere to social norms to derive their approval and companionship (Borsari and Carey 2001; Meisel and Barnett 2017). We therefore expect that behavioral confirmation will be more important for happiness in young adulthood than later in life, when identities and social networks tend to stabilize. Consequently, we expect that young adults are less happy when they cannot fulfil their need for behavioral confirmation and that this association is stronger in younger than in older age groups.

\subsubsection{Status and Happiness Across the Life Course}

Socioemotional selectivity theory asserts that younger people prioritize future-oriented goals as most of their years are likely to be ahead of them (Carstensen et al. 1999; Reed et al. 2014). Future-oriented goals include personal development, knowledge acquisition, and establishing new social contacts (Löckenhoff and Carstensen 2004, p. 1397). Knowledge and status will be useful throughout the life course and can therefore be seen as an investment (Arnett 2000, p. 69; Carstensen et al. 1999). As status goals are prioritized more by younger people, it can be expected that achieving status goals will result in more happiness for younger than for older people. Based on socioemotional selectivity theory, we thus expect a stronger relation between status fulfilment and happiness for younger age groups than for older age groups, as younger people have more time to benefit from their status investments and thus expect to be more happy once they achieve status goals. 


\subsection{The Present Study}

This paper was aimed to examine whether levels of specific social need fulfilment and their association with happiness differ between age groups. According to SPF theory, the fulfillment of different social needs along the life course can be substituted to derive the same level of happiness. Substitution implies that disharmonious need fulfilment profiles might exist, such as low affection with high confirmation and medium status, and that both the prevalence and yields of these profiles may differ across the life course. For instance, middle adulthood is characterized by a career peak and therefore profiles with high status fulfilment may be expected in this age group. Our first aim was to identify such disharmonious social need fulfilment profiles, which we expected to differ between age groups. Subsequently, we compared average levels of social need fulfilment between different age groups, as changing social contexts were expected to influence the available resources to fulfil the social needs. Third, we aimed to examine whether the relation between the fulfilment of specific social needs and happiness differs between age groups. A difference in the importance of social needs for happiness between age groups would support the tenets of socioemotional selectivity theory, whereas stable associations between social needs and happiness would support SPF theory.

\section{Methods}

\subsection{Respondents and Procedure}

This study included 11,487 adult respondents (age $18^{+}$) of the ongoing Dutch crowdsourcing study HowNutsAreTheDutch (HND; Dutch: HoeGekIsNL), who reported on the fulfilment of their social needs. ${ }^{2}$ HND allows respondents to investigate their mental health dimensions and compare themselves to others by visiting the internet platform hoegekis. nl. All procedural details are provided elsewhere (Van der Krieke et al. 2016). Respondents first completed a sociodemographic questionnaire $(n=14,489)$, after which they completed one or more topical modules (e.g., living situation, mood, or well-being). Respondents were in majority women $(67 \%)$, on average 45 years old $(S D=15)$, and higher educated (76\%). Sampling biases were intended to be accounted for by weighting our results to match demographic strata in the general Dutch population.

The HND study protocol was assessed by the Medical Ethical Committee of the University Medical Centre Groningen. The committee judged the protocol to be exempted from review by the Medical Research Involving Human Subjects Act (in Dutch: WMO) because it concerned a non-randomized open study targeted at anonymous volunteers in the general public (registration number M13.147422 and M14.160855).

\footnotetext{
${ }^{2}$ In the first week of data collection, it turned out that also people from Belgium participated. Hence, a question was added to check respondents' country. As we do not expect big differences in need fulfillment between Belgian and Dutch people, and they comprise only about $1 \%$ of the sample, we decided to keep these respondents in the study.
} 


\subsection{Measures}

\subsubsection{Social Needs}

Social needs were assessed using the short version of the SPF Index Level questionnaire (SPF-IL(s)), covering both the social and physical needs according to SPF theory (Nieboer et al. 2005). The social need subscales measure the fulfilment of affection (e.g., "Do people pay attention to you?", $\alpha=.80$ in this study), behavioral confirmation (e.g., "Do others appreciate your role in the group?", $\alpha=.66$ ) and status (e.g., "Are you known for the things you have accomplished?", $\alpha=.79$ ). All need fulfilment subscales contained three questions, which could be answered with I "never" $(=0)$, "sometimes" $(=1)$, "often" (=2), or "always" (=3) felt that way during the past 3 months (all items are provided in Supplement 1, see https://osf.io/njwuy/ for all supplements). Summing all items, the social need scales could range from 0 to 9 (affection, $M=5.83, S D=1.72$; behavioral confirmation, $M=5.82, S D=1.42$; status, $M=3.85, S D=1.70$ ). To ease interpretation and to construct interaction effects, the scores on social need fulfilment were centered around the grand mean. As an external validation, we examined correlations between the social needs and sociodemographic variables (see Supplement 1 for all details), which were in expected directions with $r=.23$ between status and income, $r=.17$ between affection and having a partner, and $r=.03$ between behavioral confirmation and church attendance, among others. These results support the construct validity of our social need operationalization.

\subsubsection{Age}

Respondents reported their year and month of birth, which was recoded into years of age. Respondents were divided into six age groups: emerging adults (18-26), young adults (age 26-40), middle-aged adults (age 40-55), late adults (55-65), young-old (65-80), and oldest-old (age 80+).

\subsubsection{Happiness}

Happiness was measured with the Happiness index (Abdel-Khalek 2006; Fordyce 2005), which reads "do you feel happy in general?". People could answer on a continuous scale from 0 (not happy at all) to 10 (very happy). The distribution of the happiness index indicates that most people report to be quite happy (Supplement 2, Figure S2.2).

\subsubsection{Control Variables}

All models were adjusted for education level and gender. Education can influence happiness both directly, for example as a resource for status (Nieboer et al. 2005), or indirectly, via income and labor status (Cuñado and de Gracia 2012). Education level was measured with eight categories ranging from "no secondary education" (=1) to "academic education" (=8). To weight our sample towards proportions in the general Dutch population according to Statistics Netherlands (www.CBS.nl), education was recoded into lower education (including education up to and including lower vocational education), medium education (intermediate vocational education up to and including higher 
secondary education), and higher education (higher professional education and academic education).

All respondents also indicated their gender $($ female $=0$, male $=1$ ). Among other reasons, gender may confound results because only men are happier and less depressed after retirement, women are not (Kim and Moen 2002). Gender differences also exist in social network constitution, as women tend to have more communication partners, whereas men tend to have better connected partners (Szell and Thurner 2013).

\subsubsection{Weights}

In our sample, men were on average older than women $(t=-18.06, p<0.001)$, with women being overrepresented in young and middle adulthood, and men in late adulthood (Supplement 2 Table S2.2). To make our results more representative for the Dutch population, we used weights to match each age group to Dutch population strata according to Statistics Netherlands using sample proportion, age, gender and education level. Exact calculations of the weights can be found in Supplement 3. Weights were restricted to the maximum of 8.00 , to prevent an excessive weight for specific individuals (see e.g., Petersen et al. 2012).

\subsubsection{Missing Values}

Less than $1 \%$ of the respondents did not report information on one of the social needs $(n=77)$, happiness $(n=58)$ or education level $(n=101)$. People who did not report on any of the social needs were excluded from all analyses, resulting in a final sample size of 11,410 respondents. Respondents who only reported on one or two social needs $(n=6)$ were included in all analyses except for the ANOVA on this specific social need. For missing values on happiness, the respondents were included in all analyses except for the ANOVA and regression analyses on happiness. Respondents who did not report education level could not be included in the weighted analyses and the regression analyses in which we control for educational level.

\subsection{Analytic Plan}

All analyses were performed in $\mathrm{R}$ ( $\mathrm{R}$ Core Team 2018), unless specified otherwise. $\mathrm{R}$ scripts are provided at https://osf.io/njwuy/. Descriptive statistics of the sample and age groups were derived first, including group means and their standard deviation (SD), and standardized mean difference $d$ between the age groups, heuristically interpreted as small from .20 to .40 , medium from .41 to .79 , and large after .80 (Borenstein 2009). The association between the social needs was examined using correlations and scatterplots. We classified correlations $(r)$ as very weak if between .00 and .29 , weak between .30 and .49 , moderate between .50 and .69 , strong between .70 and .89 , and very strong from .90 onwards (Mukaka 2012). In this study, only estimates with alpha levels below .001 will be interpreted to avoid type I errors.

Distinct social need fulfilment profiles were explored with Latent Class Analyses (LCA) in LatentGOLD (Vermunt and Magidson 2005). LCA is particularly useful for cluster analyses with a small number of items and a relatively large sample size (Eid et al. 2003). As only 16 respondents fell in the oldest-old group, this group was excluded from all cluster analyses. We fitted various types of LCA models, each with 1 up to 10 clusters. For 
each model, the number of clusters was identified by selecting the solution with the lowest BIC value. First, to examine cluster solutions for the general population, the three ordinal social need variables were clustered in the total sample and the weighted sample. Second, the same cluster analyses were performed for the five age groups separately. Third, three multi-group LCA's were performed to examine the differences between age groups in more detail.

The first multi-group LCA has an equal number of clusters across all age groups, but the model parameters may differ across the age groups. This analysis gives insight into differences in cluster solutions between the different age groups. The second multi-group LCA is constrained insofar that the association between the latent classes and the social need variables is equal for all age groups, while the cluster sizes may differ across the age groups. In this way, the interpretation of the clusters is equal across the age groups, and we could examine if certain clusters are only present in specific age groups. The last multi-group LCA was most constrained because the equality constraints of the second model were applied extended with the constraint that the cluster sizes are equal across age groups. For this model, we took the same number of clusters as we had identified in the second model. A deviance test was applied to test whether the cluster sizes were equal across age groups. The multi-group LCA's were performed using both the weighted and unweighted sample.

The differences in social need fulfilment between the age groups were examined using descriptive statistics, ANOVA analyses and pairwise t-tests. To examine whether comparisons of average social need fulfilment levels between age groups are valid, we fit measurement invariance tests (Supplement 5), which supported configural, metric, scalar, and strict invariance $(\mathrm{CFI}>.96$, RMSEA <.06; Putnick and Bornstein 2016), in line with previous reports in five independent samples (Steverink et al. 2019).

Finally, age group differences in the associations between the social needs and happiness were examined in a series of regression analyses. As the relative benefit of a social need for happiness might depend on the level of need fulfilment, it is important to control in these analyses for the average levels of need fulfilment in each age group. For example, based on social emotional selectivity theory, we expect that status is stronger associated with happiness in younger adults compared to older adults. Simultaneously, younger adults are expected to have lower levels of status fulfilment than older adults as they still have to develop skills and a career. To ensure that our interaction effects do not reflect diminishing marginal returns (the higher the need fulfilment, the lower the relative benefit), our regression models are adjusted with quadratic terms of the social needs (see Nieboer and Lindenberg 2002).

In total, four regression analyses were performed. First, the association between social need fulfilment and happiness is examined (model 1), adjusted for gender, education level, and diminishing marginal returns (model 2). Second, the association between social need fulfilment and happiness is compared between age groups via their interaction terms (age group*social need, see model 3), which was also estimated adjusted for gender, education level and diminishing marginal returns (in model 4). All regression models were repeated using sample weights to derive more generalizable conclusions.

In general, conclusions from weighted and unweighted analyses were very similar. Therefore, we mainly discuss the results from the weighted analyses. Only when the results differ, we will also discuss the results from the unweighted analyses. 

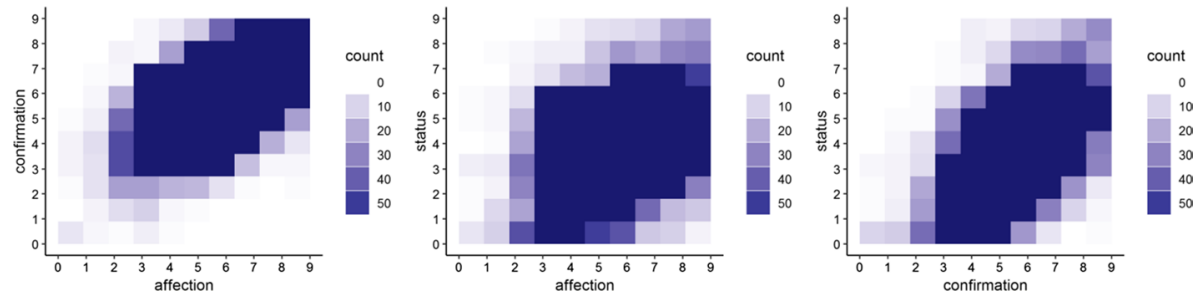

Fig. 2 Scatterplot of social needs. Count indicates how many respondents scored the corresponding values on the social needs. Dark blue indicates that more than 50 respondents scored these values, white indicates that no respondent scored these values

\section{Results}

Bivariate correlations, means, and SDs among all modeled variables, as well as descriptive statistics per age group, are provided in Supplement 2. Patterns of results were as expected, with a weak to moderate positive relationship between the social needs and happiness ( $r=.34$ to .51$)$ and between all social needs ( $r=.35$ to .58$)$.

\subsection{Patterns in Social Need Fulfilment}

To explore profiles of social need fulfilment, disharmonious combinations were examined using scatterplots and cluster analyses. Respondents seldom reported low levels of affection combined with high levels of behavioral confirmation or vice versa (Fig. 2), and no respondent experienced high levels of status and low levels of affection or confirmation. A few respondents reported low levels of status and high levels of affection or behavioral confirmation. Overall, patterns of disharmonious social need fulfilment were not found.

LCA clustering of the social needs in the total sample in general did not reveal clusters with disharmonious need fulfilment profiles. The 6-cluster model fitted the data best, given the lowest BIC value using the total sample (Supplement 4.2). People in different clusters scored generally lower or higher on all needs simultaneously, which indicates that there were no clusters of respondents with disharmonious need fulfilment profiles. These results were similar when all age groups were studied separately, which still showed clusters with lower or higher need fulfilment in general, although the number of optimal clusters differed between 4 and 6 (Supplement 4.6). Thus, in these analyses the clusters differed only in their level of the fulfillment of all needs (low to high), and not in terms of different fulfilment levels of the different needs (one low, one high). Multi-group LCA clustering of the social needs resulted mainly in clusters of generally low and high profiles of need fulfilment as well. The first multi-group LCA (equal number of clusters, with possibly different model parameters across age groups) revealed that the 4-cluster model fitted best. These 4-cluster solutions were found to be very similar for all age groups, with again only generally lower or higher scores on all social need fulfilments. The second multi-group LCA (with equal associations between latent classes and variables, with possibly varying cluster sizes across age groups) revealed that the 6-cluster model provided the best fit. Comparing these models with and without equality constraints on the cluster sizes across the age groups (using the deviance test), revealed that the models with varying cluster sizes showed a significantly better fit to the data $\left(\chi^{2}=142 ; d f=20, p<0.0001\right)$. The models with varying cluster 


\begin{tabular}{|c|c|c|c|c|c|c|}
\hline \multicolumn{7}{|c|}{$\begin{array}{l}\text { Mean levels of social need fulfilment per cluster } \\
\text { and percentages of age group members per cluster }\end{array}$} \\
\hline & & Cl. 2 & Cl. 3 & Cl. 4 & Cl. 5 & Cl. 6 \\
\hline & & & $(16 \%)$ & $(10 \%)$ & $(10 \%)$ & $(1 \%)$ \\
\hline & & & & & 2 & \\
\hline & & & & & 0 & \\
\hline & & & & & & 0. \\
\hline & & & & & & 0.02 \\
\hline & & 0.13 & 0.16 & 0.10 & 0.25 & 0.01 \\
\hline Middle adu & 0.45 & 0.27 & 0.14 & 0.10 & 0.03 & 0.01 \\
\hline & & 0 & & 0 & 0.00 & 0.01 \\
\hline foung old & 0.39 & 0.57 & 0.11 & 0.12 & 0.00 & 0.02 \\
\hline
\end{tabular}

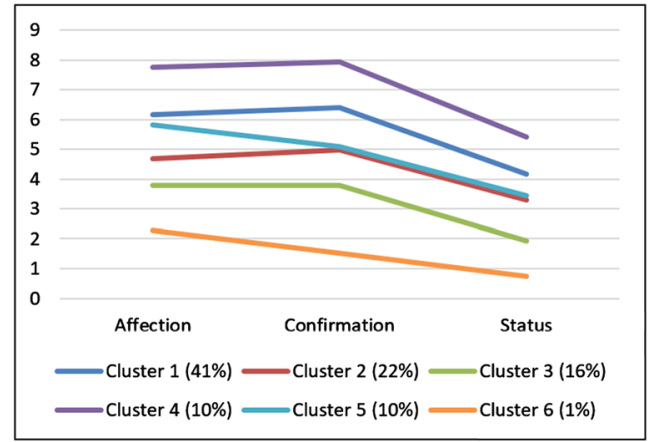

Fig. 3 Average levels of social need fulfilment per cluster. Results of best-fitting 6-cluster analysis in the unrestricted multi-group LCA (weighted). Cluster 2 consists mainly of middle adults, late adults, and the young old, while cluster 5 mainly consists of emerging and young adults. More information on the analysis can be found in the Method section

sizes are therefore interpreted. Next to low and high cluster profiles, we also found clusters with relatively higher levels of affection need fulfilment (see Fig. 3). These clusters were most common for emerging and young adults.

\subsection{Differences in Social Need Fulfilment Between Age Groups}

To examine age group differences in average social need fulfilment levels, ANOVA analyses were performed. The analyses showed significant, but small age group differences in affection $\left(F_{(5,11309)}=12.60, p<.001\right)$, behavioral confirmation $\left(F_{(5,11305)}=7.67, p<.001\right)$, and status $\left(F_{(5,11304)}=4.45, p<.001\right)$. The maximum difference between age groups was 0.48 (on a 9-points scale; see Supplement 2 for details). All effect sizes for differences in social need fulfilment between age groups were below $d=0.21$, which is typically regarded to indicate a small effect (Borenstein 2009). These results thus indicate that need fulfilment levels are highly similar between age groups (Fig. 4). Affection fulfillment levels were highest in young adulthood, and slightly higher than behavioral confirmation fulfillment levels. In middle and late adulthood, the fulfilment of behavioral confirmation was slightly higher than affection. Status fulfilment levels were slightly higher in older age groups. In sum, some age differences were observed, but the effect sizes were all very small in magnitude.

In the unweighted analyses, social need fulfilment scores were slightly higher and showed peaks for affection (about age 30) and behavioral confirmation (about age 60) (Fig. 4b). These peaks seemed to reflect the higher proportion of women in the unweighted analyses. Post-hoc tests indeed showed significant differences between women and men for affection (two-sample $t_{(7613.10)}=8.28, p<.001$ ) and status (two-sample $t_{(7212.70)}=-16.96$, $p<.001$ ), but similar levels of behavioral confirmation (two-sample $t_{(7115.40)}=-1,08$, $p<.28)$. Women reported 0.28 points higher average levels of affection fulfilment $(d=0.15)$ but 0.57 points lower average levels of status fulfilment than men $(d=-0.31$, see Fig. $4 c)$.

Figure 5 shows that the variance in need fulfilment looked similar between all age groups. Although the sample scores were highly heterogeneous, in all age groups about half of the people scored between 5 and 7 for fulfilment of affection and behavioral confirmation, and between 3 and 5 for status fulfilment. Taken together, our results suggest 
(a) Need fulfilment

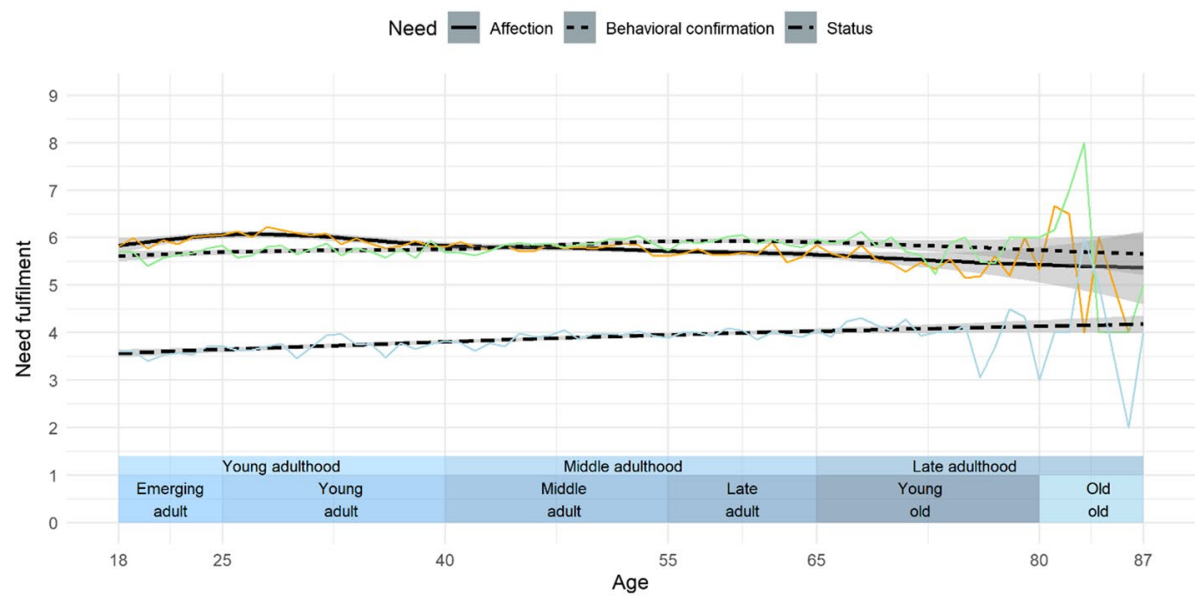

(b) Weighted (red) vs unweighted (blue) sample
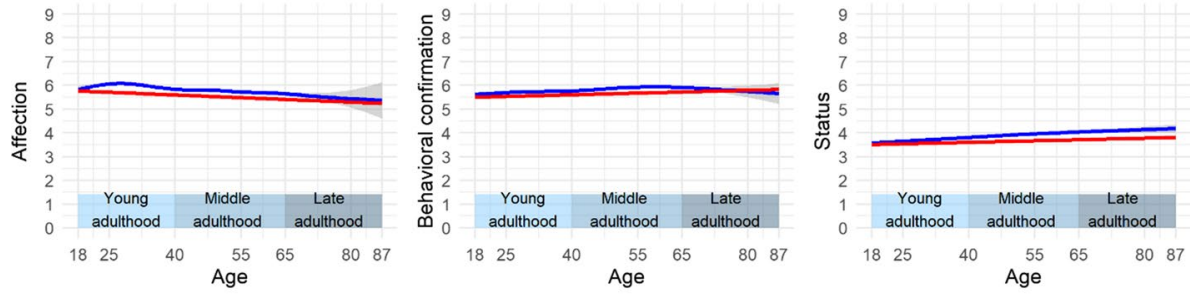

(c) Women (red) vs men (green)
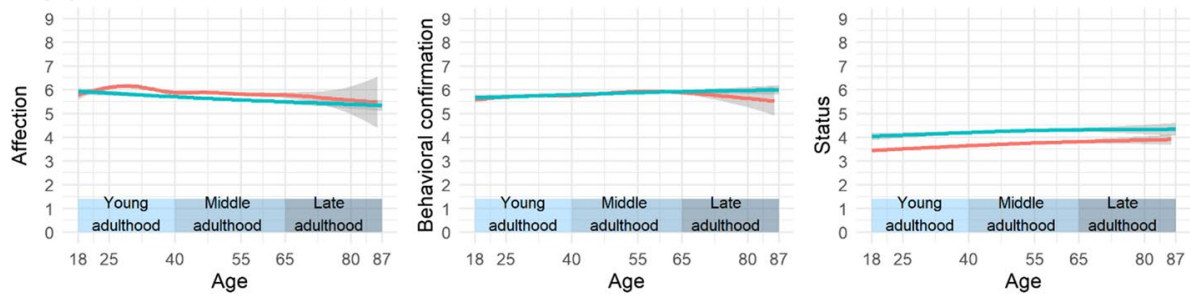

(d) Social needs and happiness
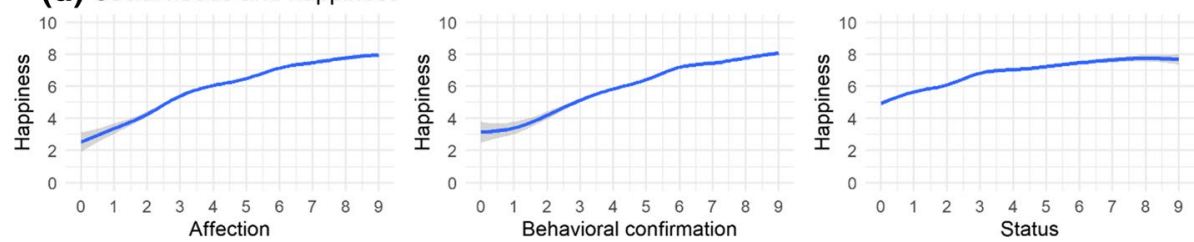

Fig. 4 Average level of need fulfilment per year of age (18-87 years). The grey area corresponds to the 95\% confidence interval. This confidence interval is much bigger for the oldest-old, which reflects the smaller sample size at high ages

that the difference in need-fulfilment between age-groups were marginal at best, and that throughout the entire life course, levels of affection and confirmation fulfilment were higher than status fulfilment levels. 


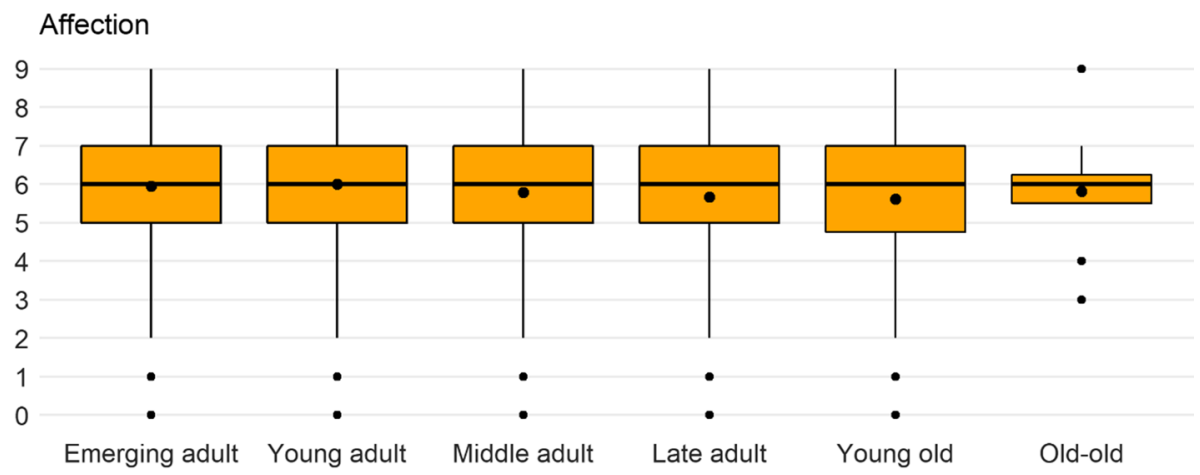

Behavioral confirmation
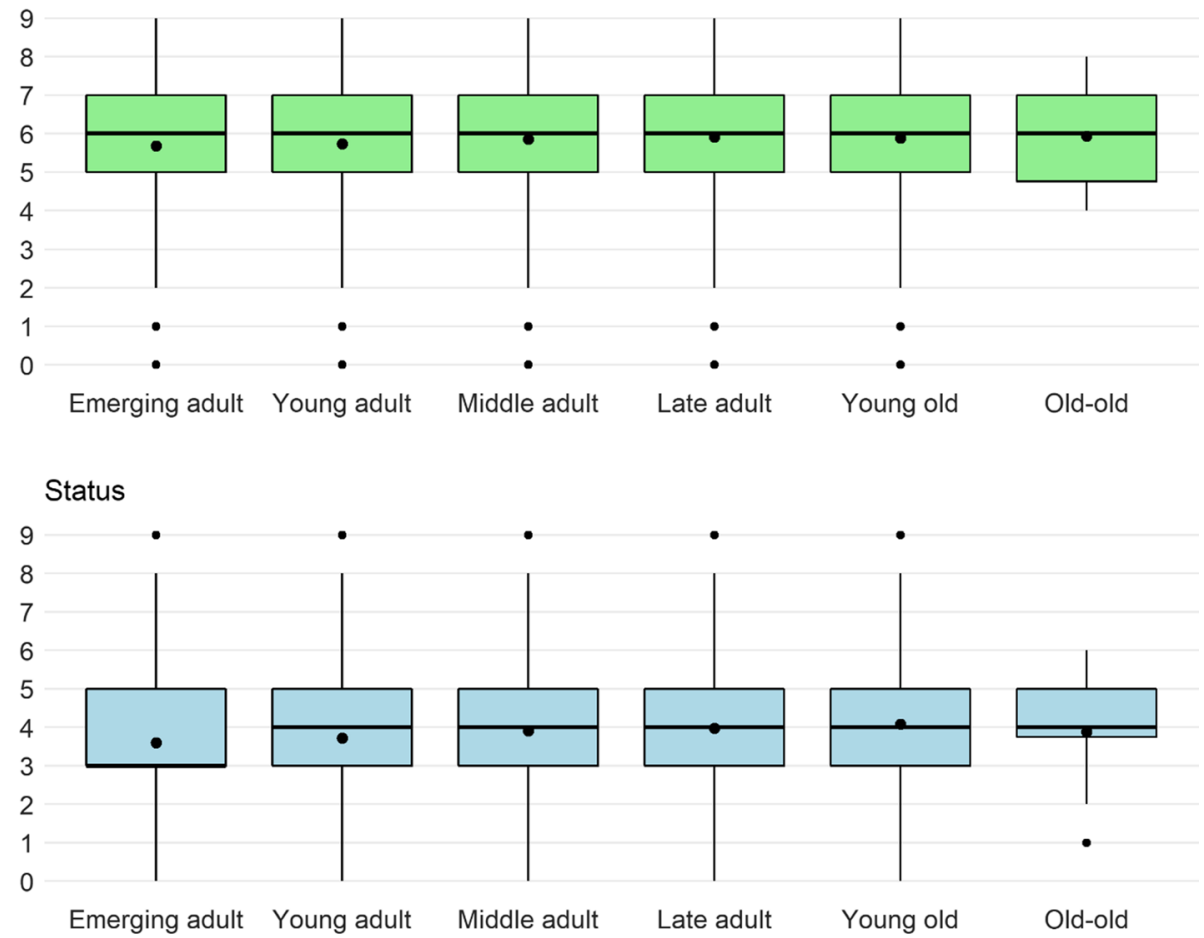

Fig. 5 Boxplots of the social needs per age group. The black dots in the middle indicate the means

\subsection{Regression Analyses}

Age group differences in the relationship between social needs fulfilment and happiness were examined by using regression analyses. The regression models are presented in Table 2. The fulfilment of each social need was associated with happiness and combined they explained up to $31 \%$ of the total variance in happiness (see Model 1). The effect sizes of social needs were predominantly independent from gender and educational level (see Model 2), even though lower education was associated with less happiness. Controls for 
Table 2 Regression analyses on happiness. Weighted by age, gender and education level $(\mathrm{N}=11,386)$

\begin{tabular}{|c|c|c|c|c|c|c|c|c|}
\hline \multirow[t]{2}{*}{ Pred. variable } & \multicolumn{2}{|l|}{ Model 1} & \multicolumn{2}{|l|}{ Model 2} & \multicolumn{2}{|l|}{ Model 3} & \multicolumn{2}{|l|}{ Model 4} \\
\hline & $b$ & $(\mathrm{SE})$ & $b$ & (SE) & $b$ & (SE) & $B$ & (SE) \\
\hline Intercept & $6.83^{* * *}$ & $(0.01)$ & $6.90 * * *$ & $(0.03)$ & $6.50 * * *$ & $(0.05)$ & $6.49^{* * * *}$ & $(0.05)$ \\
\hline Affection & $0.35^{* * *}$ & $(0.01)$ & $0.32 * * *$ & $(0.01)$ & $0.33 * * *$ & $(0.01)$ & $0.41^{* * *}$ & $(0.02)$ \\
\hline Confirmation & $0.23 * * *$ & $(0.01)$ & $0.21 * * *$ & $(0.01)$ & $0.19 * * *$ & $(0.01)$ & $0.16^{* * * *}$ & $(0.03)$ \\
\hline Status & $0.13^{* * *}$ & $(0.01)$ & $0.11 * * *$ & $(0.01)$ & $0.11 * * *$ & $(0.01)$ & $0.09^{* * *}$ & $(0.02)$ \\
\hline Gender (women) & & & 0.03 & $(0.03)$ & 0.01 & $(0.03)$ & 0.02 & $(0.03)$ \\
\hline Medium education $^{1}$ & & & $0.15^{*}$ & $(0.04)$ & $0.24 * * *$ & $(0.04)$ & $0.25^{* * *}$ & $(0.04)$ \\
\hline Higher education & & & $0.25 * * *$ & $(0.04)$ & $0.31 * * *$ & $(0.04)$ & $0.32^{* * *}$ & $(0.04)$ \\
\hline Affection $^{2}$ & & & $-0.03 * * *$ & $(0.00)$ & $-0.03^{* * *}$ & $(0.00)$ & $-0.03^{* * *}$ & $(0.00)$ \\
\hline Confirmation $^{2}$ & & & $-0.03 * * *$ & $(0.00)$ & $-0.03^{* * *}$ & $(0.00)$ & $-0.03^{* * *}$ & $(0.00)$ \\
\hline Status $^{2}$ & & & $-0.02 * * *$ & $(0.00)$ & $-0.02 * * *$ & $(0.00)$ & $-0.02 * * *$ & $(0.00)$ \\
\hline Young adult $^{3}(n=2714)$ & & & & & $0.21 * * *$ & $(0.05)$ & $0.24 * * *$ & $(0.05)$ \\
\hline $\begin{array}{l}\text { Middle-aged adult } \\
\quad(\mathrm{n}=3700)\end{array}$ & & & & & $0.30 * * *$ & $(0.05)$ & $0.31 * * *$ & $(0.05)$ \\
\hline Late adult $(\mathrm{n}=2567)$ & & & & & $0.43 * * *$ & $(0.05)$ & $0.43^{* * *}$ & $(0.05)$ \\
\hline Young-old $(\mathrm{n}=887)$ & & & & & $0.68 * * *$ & $(0.05)$ & $0.70^{* * *}$ & $(0.05)$ \\
\hline Oldest-old $(\mathrm{n}=16)$ & & & & & $0.91 * * *$ & $(0.16)$ & $0.92^{* * *}$ & $(0.16)$ \\
\hline Affection*Young adult & & & & & & & $-0.14 * * *$ & $(0.03)$ \\
\hline Affection*Middle adult & & & & & & & $-0.07 *$ & $(0.03)$ \\
\hline Affection*Late adult & & & & & & & $-0.14 * * *$ & $(0.03)$ \\
\hline Affection*Young-old & & & & & & & -0.05 & $(0.03)$ \\
\hline Affection*Oldest-old & & & & & & & $-0.49 * * *$ & $(0.14)$ \\
\hline Confirmation*Young adult & & & & & & & $0.10^{* *}$ & $(0.04)$ \\
\hline Confirmation*Middle adult & & & & & & & 0.02 & $(0.04)$ \\
\hline Confirmation*Late adult & & & & & & & $0.11^{* *}$ & $(0.04)$ \\
\hline Confirmation*Young-old & & & & & & & -0.05 & $(0.04)$ \\
\hline Confirmation*Oldest-old & & & & & & & -0.23 & $(0.12)$ \\
\hline Status*Young adult & & & & & & & 0.04 & $(0.03)$ \\
\hline Status*Middle adult & & & & & & & 0.00 & $(0.03)$ \\
\hline Status*Late adult & & & & & & & 0.01 & $(0.03)$ \\
\hline Status*Young-old & & & & & & & 0.05 & $(0.03)$ \\
\hline Status*Oldest-old & & & & & & & 0.00 & $(0.15)$ \\
\hline $\mathrm{R}^{2 \mathrm{a}}$ & $.30 * * *$ & & $.32 * * *$ & & $.33 * * *$ & & $.34 * * *$ & \\
\hline
\end{tabular}

${ }^{1}$ Reference group is lower education; $* * * p<.001, * * p<.01, * p<.05$

${ }^{2}$ Quadratic term

${ }^{3}$ Reference group is emerging adults $(n=1502)$

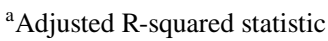

diminishing marginal returns reached significance (Model 2), which means that at a higher level of social need fulfilment, the relative effect of additional need fulfillment on happiness decreased. However, the effect size of these diminishing marginal returns was of little importance in the effect of social needs on happiness. The older age groups were on average happier than the younger age groups (Model 3). 


Affection
Confirmation
Status
Women
Secondary education
Higher education
Affection^2
Confirmation`2
Status^2
Young adult
Middle-aged adult
Late adult
Young old
Oldest-old
Affection YA
Affection MA
Affection LA
Affection YO
Affection OO
Confirmation YA
Confirmation MA
Confirmation LA
Confirmation YO
Confirmation OO
Status YA
Status MA
Status LA
Status YO
Status OO

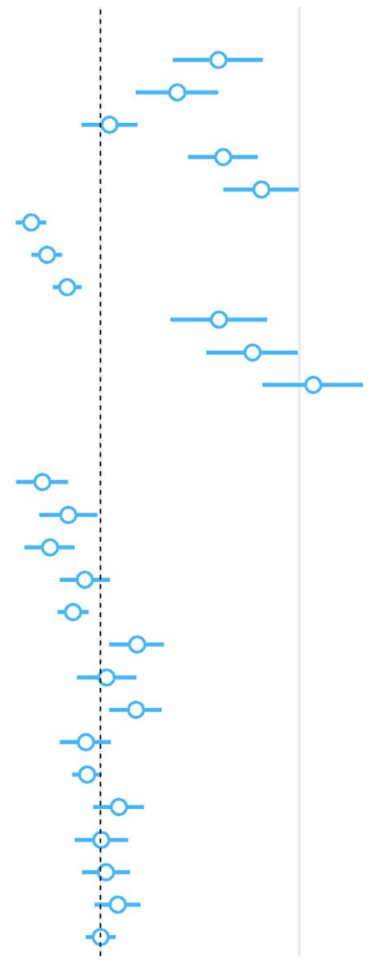

0.0

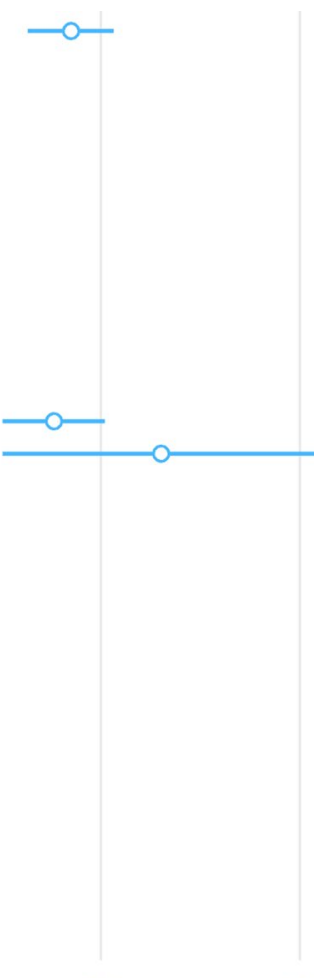

0.8 1.2

Estimate

Fig. 6 Visualization of effects from the regression analysis (Model 4)

The interaction effects did not show a clear picture (Model 4, Fig. 6). With all 15 interaction terms included ( 3 needs $* 5$ age groups), the model explained only 1 percentage point more variance in happiness. Thus, the combined effect of all interactions was very small. The separate significant interaction effects were small as well, and lost statistical significance in the unweighted analyses (see Supplement 6). Overall, we did not find consistent support for any of our hypotheses on age group differences in the relation between need fulfilment and happiness.

\section{Discussion}

In this study we aimed to explore (a) whether disharmonious profiles of social need fulfilment could be identified, (b) whether people from different age groups differed in fulfilment of affection, behavioral confirmation and status needs and (c) in the relative importance of social need fulfilment for happiness. Our study yielded three key observations: (1) Clusters of specific profiles of social need fulfilment could not be identified, beyond general lower or higher scores; (2) We observed a remarkable stability in average social need fulfilment across age groups; (3) The importance of fulfilling specific social needs for happiness did not differ along the life course. Our key conclusion is therefore that some 
people seem more capable than others to fulfil their social needs in general, regardless of chronological age. These observations are now discussed in detail below.

\subsection{Profiles of Social Needs}

Our first aim was to identify potential distinct profiles of social need fulfilment, which we expected to follow an age gradient. Contrary to our expectations, our models yielded need fulfilment profiles with lower or higher fulfilment of all social needs. Disharmonious social need fulfilment patterns (i.e., one need high and one low) were rare in our sample. Nonetheless, in one of our models, some clusters showed relatively higher levels of affection, primarily in emerging and young adults. If there would be a difference in social need fulfilment related to age groups, then our results point towards higher affection need fulfilment in younger people. Substitution between social needs, as suggested by SPF theory, is not observed in our age groups. Our results seem to point at a distinction between people who are able to fulfil all three social needs, and people who have more trouble doing so in general.

Substitution does seem to happen in some people, but only in one direction, as some respondents reported low status fulfillment but high affection and behavioral confirmation. This observation is in line with SPF theory which predicts that status is more difficult to obtain than affection and therefore status is more likely to be substituted for affection than vice versa (e.g., Steverink 2001).

\subsection{The Absence of Life Course Differences in Social Need Fulfilment}

Our second aim was to compare average levels of social need fulfilment between age groups, as we expected differences following life course differences in social roles, resources, and contexts. The expected patterns of need fulfilment were not found. Overall, differences between age groups were very small (all below $d=0.21$ ). In young adulthood, slightly lower levels of behavioral confirmation fulfilment and slightly higher levels of affection fulfilment were reported than in middle and late adulthood. Older age groups reported slightly higher levels of status fulfilment, and contrary to our expectations, we observed no difference in status fulfilment between the age groups before and after the average retirement age.

Overall, few differences in the fulfilment of social needs were observed between age groups, contrary to our expectations. One possible explanation is that when people report on the fulfilment of social needs, they use their age group as frame of reference when making comparisons (Pérez-Asenjo 2011). For example, young adults might feel that, compared to other people of the same age, they have relatively high levels of status, while they might have answered differently if they would have thought of a comparison with older adults (cf. Suls et al. 2002).

The observation that the fulfilment of status needs did not decline after the retirement age does not align with previous research (e.g., Steverink and Lindenberg 2006). This stability of status after retirement might reflect the overrepresentation of higher educated people in our sample, despite our weighted analyses. Possibly, people with high education maintain their status (e.g., as a doctor or professor), while lower educated people lose their status (e.g., as a construction worker or nurse). In line with this idea, a previous study of retiring people showed little difference in happiness (operationalized as life satisfaction) in higher educated retirees but a decline after retirement in retirees with lower education 
(Wetzel et al. 2016). Future studies could explore this status effect in more detail, and examine the frame-of-reference hypothesis.

Although we did not find major differences in social need fulfilment between age groups, we did find differences between men and women. The peaks for affection (about age 30) and behavioral confirmation (about age 60) disappeared in the weighted estimates, seemingly due to the high proportion of women in the unweighted analyses, with diluted effects in men. Women reported slightly higher levels of affection than men, but significantly lower levels of status, which has previously been observed by Steverink et al. (2019). Future studies might expand upon our results and investigate whether gender differences also influence the ways in which people fulfil their needs.

The small age differences in need fulfilment over the life course point to individual rather than age-related effects on social need fulfilment. Individuals are likely to differ considerably in the resources they have available to fulfill needs, which can be corroborated by the large range in need fulfilment within age groups. Importantly, the observed stability in need fulfilment levels across the life course could indicate that people primarily differ in the ways in which they fulfill their needs, leaving overall need fulfilment levels unchanged. Consequently, people first compensate for losses in affection by seeking alternative means to fulfil this same need (in line with SPF theory) and only after having lost many resources, people start to substitute between needs, such as compensating status loss by increasing the production of affection. It may be that our sample, despite age differences, is too homogeneous in health and other resources to observe consistent need substitution.

To summarize, based on our models, it is likely that age groups do not differ substantively in their average need fulfilment, but only in the ways they derive the same degree of need fulfilment, which we could not test. Future studies can examine how different people fulfil their needs for affection, behavioral confirmation, and status in more detail. Ideally, these studies also sample different populations, as the Dutch are among the happiest populations on earth (Helliwell et al. 2019).

\subsection{Stability in Social Need Fulfilment and Happiness Across Age Groups}

Our third aim was to explore whether and how age groups differed in the relative importance of the fulfilment of the three different social needs for their happiness. Our key observation is that the fulfillment of all three social needs seems to be equally important for happiness across the adult life course. Some significant interactions were found for affection and behavioral confirmation $\left(\Delta \mathrm{R}^{2}<.01\right)$, but these effects were only found in the weighted analysis and are potentially due to the truncated values for the very high weights. Indeed, when examining the same question with merged age-groups (young [age 18-40], middle [age 40-65], and old [age 65 $5^{+}$adults), no significant interactions occurred (Supplement 7).

SPF theory asserts that all three social needs are universal, and thus produce wellbeing, regardless of age. This was supported by our regression models. These observations are not entirely in line with socioemotional selectivity theory insofar that older adults are assumed to derive more well-being from close relations (i.e., affection) than younger adults, while the latter are assumed to get more well-being from investing in long term goals (including status). However, according to both SPF and socioemotional selectivity theory, the strongest effects may be expected at the highest ages, when resources seriously decline (SPF) and time horizon shortens (SST). Our pool of respondents, however, comprised only sixteen people over 80 years old. Perhaps the effects of the shifting importance of specific needs on happiness may not have 
been visible in the present sample, because the decline of fulfilment of social needs is better observed in the oldest-old. Furthermore, we cannot rule out that some people answered the questions on need fulfilment relative to their peers (e.g., "My status is higher than the status of my peers"), while others may have kept an ideal situation in mind, regardless of age (e.g., "My status is lower than I wish it were"). Future studies should explore possible changes in social need fulfilment in the oldest-old and frameof-reference effects in more detail.

\subsection{Limitations}

As we compared people from different age groups, cohort effects might have played a role in these results. Our society, social roles, and resources changed significantly for different generations over the past century (e.g., Mitchell 2006; Pilcher 1994). Our measures of measurement invariance, however, indicated that people from different age groups answered the items of the scales on social need fulfilment in a similar way, and this has recently also been confirmed in five independent samples with various age groups (Steverink et al. 2019). Therefore, we can assume that the age groups interpreted the questions about need fulfilment similarly. Although developmental questions in principle require a within-individual longitudinal design, such long-span birthcohort studies are scarce. Moreover, changes in social and economic circumstances at the moment of measurement renders conclusions about young adults obsolete once this sample became old, which also impedes inter-generational comparisons. Although we did establish measurement invariance, some cohort effects cannot be ruled out. Due to the cross-sectional design, reversed causality (or third factors) might also play a role, as happier people might be more able to fulfil their social needs.

Furthermore, the sample used here is not completely representative for the Dutch population. Even though our selected sample showed a wide range of scores and we weighted our results based on population strata (see method section), selection effects may have played a role. For instance, only people willing to spend their free time and who could find the website of hoegekis.nl filled out the online questionnaires. About 2.5 million people in the Netherlands (15\%) lack the reading and writing skills to participate (Algemene Rekenkamer 2016). Still, such selection effects may attenuate the estimated effect sizes at worst, but not alter the underlying processes and our main conclusions. Replicating these results in different and less advantaged samples could strengthen the claim that age groups are strikingly similar in need fulfilment.

Finally, happiness can be measured in various ways (Linton et al. 2016). Although the single-question happiness scale has been well-validated (Abdel-Khalek 2006), different results could be obtained with different measurements of happiness. For example, status fulfilment previously showed stronger associations with positive affect, and behavioral confirmation with negative affect, while affection is more associated with life satisfaction (e.g., Nieboer et al. 2005; Steverink and Lindenberg 2006; Steverink et al. 2019). Different happiness measures might also lead to different outcomes for different countries, as for example the fulfilment of social needs is higher correlated with positive emotions than life evaluation in Northern Europe, but not in Africa (Tay and Diener 2011). Using a different measure of happiness might thus result in slightly different results. 


\section{Conclusion}

Overall, we found no substantive differences between age groups in their fulfillment of their affection, behavioral confirmation or status needs. Moreover, because of the large sample size, it is unlikely that there will be large differences in levels of need fulfilment between age groups in the Dutch population. If there are differences, they are most likely to be small and subtle. On the other hand, a large variation in need fulfilment is found within age groups, with a similar distribution of variability for all needs and age groups. ${ }^{3}$ Individual differences in need fulfilment seem more important for happiness than age differences at the group level (cf. Fisher et al. 2018). Perhaps when life phases are examined in terms of developmental age (via life experiences including having a partner, children, or job) instead of chronological age differences, group differences in need fulfilment may become visible. There are thus still mysteries to be solved, and although chronological age does not explain differences in fulfilment of the three social needs on the group level, living through the life course while the availability of resources changes may still influence specific social need fulfilments in individual life trajectories.

Acknowledgements We thank all respondents and collaborators of the HowNutsAreTheDutch study for their contribution. We also thank Marieke Timmerman for sharing her expertise on cluster analyses and reviewer 2 for their constructive comments. This research was supported by grants from the Dutch Research Council (NWO) received by Peter de Jonge (Vici no. 91812607), Bertus Jeronimus (016.Veni.195.405), and Gerine Lodder (016.Veni.195.186).

Open Access This article is licensed under a Creative Commons Attribution 4.0 International License, which permits use, sharing, adaptation, distribution and reproduction in any medium or format, as long as you give appropriate credit to the original author(s) and the source, provide a link to the Creative Commons licence, and indicate if changes were made. The images or other third party material in this article are included in the article's Creative Commons licence, unless indicated otherwise in a credit line to the material. If material is not included in the article's Creative Commons licence and your intended use is not permitted by statutory regulation or exceeds the permitted use, you will need to obtain permission directly from the copyright holder. To view a copy of this licence, visit http://creativecommons.org/licenses/by/4.0/.

\section{References}

Abdel-Khalek, A. M. (2006). Measuring happiness with a single-item scale. Social Behavior and Personality: An International Journal, 34(2), 139-150. https://doi.org/10.2224/sbp.2006.34.2.139.

Algemene Rekenkamer. (2016). Aanpak van laaggeletterdheid. https:/www.rekenkamer.nl/publicaties/ rapporten/2016/04/20/aanpak-van-laaggeletterdheid.

Anant, S. S. (1967). Belongingness and mental health: Some research findings. Acta Psychologica, 26, 391396. https://doi.org/10.1016/0001-6918(67)90035-2.

Arnett, J. J. (2000). Emerging adulthood: A theory of development from the late teens through the twenties. American Psychologist, 55(5), 469-480. https://doi.org/10.1037/0003-066X.55.5.469.

Baumeister, R. F., \& Leary, M. R. (1995). The need to belong: Desire for interpersonal attachments as a fundamental human motivation. Psychological Bulletin, 117(3), 497. https://doi. org/10.1037/0033-2909.117.3.497.

Belsky, J., \& Rovine, M. (1984). Social-network contact, family support, and the transition to parenthood. Journal of Marriage and the Family, 46(2), 455-462. https://doi.org/10.2307/352477.

Bernardi, L., \& Klärner, A. (2014). Social networks and fertility. Demographic Research, 30, 641-669. https ://www.jstor.org/stable/26348213.

\footnotetext{
${ }^{3}$ Except for the oldest-old $(80+)$, but since this group only consisted of 16 individuals these results can be ignored.
} 
Borenstein, M. (2009). Effect sizes for continuous data. In H. Cooper, L.V. Hedges, \& J.C. Valentine (Eds.), The handbook of research synthesis and meta-analysis (pp. 221-235). https://www.jstor.org/stabl e/10.7758/9781610441384.

Borsari, B., \& Carey, K. B. (2001). Peer influences on college drinking: A review of the research. Journal of Substance Abuse, 13(4), 391-424. https://doi.org/10.1016/S0899-3289(01)00098-0.

Bos, E. H., Snippe, E., de Jonge, P., \& Jeronimus, B. F. (2016). Preserving subjective wellbeing in the face of psychopathology: Buffering effects of personal strengths and resources. PLOS ONE, 11(3), e0150867. https://doi.org/10.1371/journal.pone.0150867.

Brody, E. M. (2004). Women in the middle: Their parent care years (2nd ed.). New York, NY: Springer.

Buote, V. M., Pancer, S. M., Pratt, M. W., Adams, G., Birnie-Lefcovitch, S., Polivy, J., et al. (2007). The importance of friends: Friendship and adjustment among 1st-year university students. Journal of Adolescent Research, 22(6), 665-689. https://doi.org/10.1177/0743558407306344.

Carstensen, L. L. (1992). Social and emotional patterns in adulthood: Support for socioemotional selectivity theory. Psychology and Aging, 7(3), 331-338. https://doi.org/10.1037/0882-7974.7.3.331.

Carstensen, L. L., Isaacowitz, D. M., \& Charles, S. T. (1999). Taking time seriously: A theory of socioemotional selectivity. American Psychologist, 54(3), 165-181. https://doi. org/10.1037/0003-066X.54.3.165.

Cramm, J. M., Hartgerink, J. M., De Vreede, P. L., Bakker, T. J., Steyerberg, E. W., Mackenbach, J. P., et al. (2012). The relationship between older adults' self-management abilities, well-being and depression. European Journal of Ageing, 9(4), 353. https://doi.org/10.1007/s10433-012-0237-5.

Cuñado, J., \& de Gracia, F. P. (2012). Does education affect happiness? Evidence for Spain. Social Indicators Research, 108(1), 185-196. https://doi.org/10.1007/s11205-011-9874-x.

Deci, E., \& Ryan, R. (2008). Self-determination theory: A macrotheory of human motivation, development, and health. Canadian Psychology, 49(3), 182-185. https://doi.org/10.1037/a0012801.

Diener, E., Oishi, S., \& Lucas, R. (2009). Subjective well-being: The science of happiness and life satisfaction. In S. J. Lopez \& C. R Snyder (Eds.), The Oxford handbook of positive psychology. Oxford: Oxford University Press. http://www.oxfordhandbooks.com/view/1.1093/oxfordhb/9780195187 243.001.0001/oxfordhb-9780195187243-e-017.

Dutch Ministry of Education, Culture and Science. (2013). Kerncijfers 2008-2012. https://www.rijksoverh eid.nl/documenten/jaarverslagen/2013/05/08/ocw-kerncijfers-2008-2012.

Dykstra, P. A. (2009). Older adult loneliness: Myths and realities. European Journal of Ageing, 6(2), 91. https://doi.org/10.1007/s10433-009-0110-3.

Ebner, N. C., Freund, A. M., \& Baltes, P. B. (2006). Developmental changes in personal goal orientation from young to late adulthood: From striving for gains to maintenance and prevention of losses. Psychology and Aging, 21, 664-678. https://doi.org/10.1037/0882-7974.21.4.664.

Eid, M., Langeheine, R., \& Diener, E. (2003). Comparing typological structures across cultures by multigroup latent class analysis: A primer. Journal of Cross-Cultural Psychology, 34(2), 195-210. https:// doi.org/10.1177/0022022102250427.

Erikson, E. H., \& Erikson, J. M. (1998). The life cycle completed (extended version). New York, NY: Norton \& Company.

Fisher, A. J., Medaglia, J. D., \& Jeronimus, B. F. (2018). Lack of group-to-individual generalizability is a threat to human subjects research. Proceedings of the National Academy of Sciences, 115(27), E6106E6115. https://doi.org/10.1073/pnas.1711978115.

Fordyce, M. W. (2005). A review of research on the happiness measures: A sixty second index of happiness and mental health. Social Indicators Research, 20(4), 355-381. https://doi.org/10.1007/BF00302333.

Gorchoff, S. M., John, O. P., \& Helson, R. (2008). Contextualizing change in marital satisfaction during middle age. Psychological Science, 19(11), 1194. https://doi.org/10.1111/j.1467-928.2008.02222.x.

Hall, J. P., Shartzer, A., Kurth, N. K., \& Thomas, K. C. (2017). Effect of Medicaid expansion on workforce participation for people with disabilities. American Journal of Public Health, 107(2), 262-264. https:// doi.org/10.2105/AJPH.2016.303543.

Helliwell, J., Layard, R., \& Sachs, J. (2019). World happiness report 2019, New York (NY): Sustainable Development Solutions Network. https://worldhappiness.report/ed/2019/.

Holt-Lunstad, J., Smith, T. B., Baker, M., Harris, T., \& Stephenson, D. (2015). Loneliness and social isolation as risk factors for mortality: A meta-analytic review. Perspectives on Psychological Science, 10(2), 227-237. https://doi.org/10.1177/1745691614568352.

Huxhold, O., Fiori, K. L., \& Windsor, T. D. (2013). The dynamic interplay of social network characteristics, subjective well-being, and health: The costs and benefits of socio- emotional selectivity. Psychology and Aging, 28(1), 3-16. https://doi.org/10.1037/a0030170. 
Jones, G., O’Sullivan, A., \& Rouse, J. (2006). Young adults, partners and parents: Individual agency and the problems of support. Journal of Youth Studies, 9(4), 375-392. https://doi.org/10.1080/1367626060 0914374.

Kim, J. E., \& Moen, P. (2002). Retirement transitions, gender, and psychological well-being: A life-course, ecological model. The Journals of Gerontology Series B: Psychological Sciences and Social Sciences, 57(3), 212-222. https://doi.org/10.1093/geronb/57.3.P212.

Klärner, A., Keim, S., \& Von der Lippe, H. (2016). Social network dynamics in the course of family formation: Results from a mixed-methods longitudinal study. International Review of Social Research, 6(4), 245-255. https://doi.org/10.1515/irsr-2016-0026.

Lachman, M. E. (2004). Development in midlife. Annual Review of Psychology, 55, 305-331. https://doi. org/10.1146/annurev.psych.55.090902.141521.

Lindenberg, S. (1996). Continuities in the theory of social production functions. In H. Ganzeboom \& S. Lindenberg (Eds.), Verklarende Sociologie: Opstellen voor Reinhard Wippler (pp. 169-184). Amsterdam: Thela Thesis.

Lindenberg, S. (2013). Social rationality, self-regulation and well-being: The regulatory significance of needs, goals, and the self. In R. Wittek, T. Snijders, \& V. Nee (Eds.), The handbook of rational choice social research (pp. 72-113). Stanford, CA: Stanford University Press.

Linton, M. J., Dieppe, P., \& Medina-Lara, A. (2016). Review of 99 self-report measures for assessing well-being in adults: Exploring dimensions of well-being and developments over time. British Medical Journal Open, 6(7), e010641. https://doi.org/10.1136/bmjopen-2015-010641.

Löckenhoff, C. E., \& Carstensen, L. L. (2004). Socioemotional selectivity theory, aging, and health: The increasingly delicate balance between regulating emotions and making tough choices. Journal of Personality, 72(6), 1395-1424. https://doi.org/10.1111/j.1467-6494.2004.00301.x.

Luthar, S. S., \& Ciciolla, L. (2016). What it feels like to be a mother: Variations by children's developmental stages. Developmental Psychology, 52(1), 143-154. https://doi.org/10.1037/dev0000062.

Marceau, K., Ram, N., \& Susman, E. J. (2015). Development and lability in the parent-child relationship during adolescence: Associations with pubertal timing and tempo. Journal of Research on Adolescence, 25(3), 474-489. https://doi.org/10.1111/jora.12139.

Maslow, A. H. (1943). A theory of human motivation. Psychological Review, 50(4), 370-396. https:// doi.org/10.1037/h0054346.

Max-Neef, M. (1992). Development and human needs. In P. Ekins \& M. Max-Neef (Eds.), Real life economics (pp. 197-214). London: Routledge.

McClelland, D. C. (1985). Human motivation. Glenview, IL: Scott, Foresman.

Medvedev, O. N., \& Landhuis, C. E. (2018). Exploring constructs of well-being, happiness and quality of life. Peer J, 6, e4903. https://doi.org/10.7717/peerj.4903.

Meisel, M. K., \& Barnett, N. P. (2017). Protective and risky social network factors for drinking during the transition from high school to college. Journal of Studies on Alcohol and Drugs, 78(6), 922-929. https://doi.org/10.15288/jsad.2017.78.922.

Mitchell, B. (2006). The boomerang age. New York, NY: Routledge. https://doi.org/10.4324/97813 15131085.

Morrison, E. W. (2002). Newcomers' relationships: The role of social network ties during socialization. Academy of Management Journal, 45(6), 1149. https://doi.org/10.2307/3069430.

Mukaka, M. M. (2012). A guide to appropriate use of correlation coefficient in medical research. Malawi Medical Journal, 24, 69-71. https://www.ncbi.nlm.nih.gov/pmc/articles/PMC3576830.

Mund, M., Jeronimus, B. F., \& Neyer, F. J. (2018). Personality and social relationships: As thick as thieves. In C. Johansen (Ed.), Personality and disease (pp. 153-183). Academic Press. http://doi. org/10.1016/B978-0-12-805300-3.00009-8.

Nieboer, A. \& Lindenberg, S. (2002). Substitution, buffers and subjective well-being: A hierarchical approach. In E. Gullone \& R.A. Cummins (Eds.), The universality of subjective wellbeing indicators (pp. 175-189). Social Indicators Research Book Series, Volume 16. Dordrecht: Springer. http://doi.org/10.1007/978-94-010-0271-4_10.

Nieboer, A., Lindenberg, S., Boomsma, A., \& Bruggen, A. C. V. (2005). Dimensions of well- being and their measurement: The SPF-IL scale. Social Indicators Research, 73(3), 313-353. https://doi. org/10.1007/s11205-004-0988-2.

Ormel, J., Lindenberg, S., Steverink, N., \& Verbrugge, L. M. (1999). Subjective well-being and social production functions. Social Indicators Research, 46(1), 61-69. https://doi.org/10.1023/A:10069 07811502.

Ormel, J., VonKorff, M., Jeronimus, B. F., \& Riese, H. (2017). Set-point theory and personality development: Reconciliation of a paradox. In J. Specht (Ed.), Personality development across the life course (pp. 117-137). Amsterdam: Elsevier. http://doi.org/10.1016/B978-0-12-804674-6.00009-0. 
Oswald, D. L., \& Clark, E. M. (2003). Best friends forever?: High school best friendships and the transition to college. Personal Relationships, 10(2), 187-196. https://doi.org/10.1111/1475-6811.00045.

Park, K. (2002). Stigma management among the voluntarily childless. Sociological Perspectives, 45(1), 21-45. https://doi.org/10.1525/sop.2002.45.1.21.

Pérez-Asenjo, E. (2011). If happiness is relative, against whom do we compare ourselves? Implications for labour supply. Journal of Population Economics, 24(4), 1411-1442. https://doi.org/10.1007/ s00148-010-0322-z.

Petersen, M. L., Porter, K. E., Gruber, S., Wang, Y., \& Van Der Laan, M. J. (2012). Diagnosing and responding to violations in the positivity assumption. Statistical Methods in Medical Research, 21(1), 31-54. https://doi.org/10.1177/0962280210386207.

Pilcher, J. (1994). Mannheim's sociology of generations: An undervalued legacy. British Journal of Sociology. https://doi.org/10.2307/591659.

Putnick, D. L., \& Bornstein, M. H. (2016). Measurement invariance conventions and reporting: The state of the art and future directions for psychological research. Developmental Review, 41, 71-79. https ://doi.org/10.1016/j.dr.2016.06.004.

R Core Team. (2018). R: A language and environment for statistical computing. R Foundation for Statistical Computing. Vienna, Austria. https://www.R-project.org.

Reed, A. E., Chan, L., \& Mikels, J. A. (2014). Meta-analysis of the age-related positivity effect: Age differences in preferences for positive over negative information. Psychology and Aging, 29(1), 1-15. https://doi.org/10.1037/a0035194.

Rözer, J., Poortman, A. R., \& Mollenhorst, G. (2017). The timing of parenthood and its effect on social contact and support. Demographic Research, 36, 1889-1916. https://doi.org/10.4054/DemRe s.2017.36.62.

Sigelman, C. K., \& Rider, E. A. (2018). Life-span human development (9e ed.). Australia: Cengage Learning.

Statistics Netherlands. (2004). Empty-nest moeders. https://www.cbs.nl/achtergrond/2004/23/empty -nest-moeders.

Statistics Netherlands. (2015). Relatievorming van twintigers. https://www.cbs.nl/nl-nl/achtergron d/2015/10/relatievorming-van-twintigers.

Statistics Netherlands. (2017). Arbeidsdeelname; Kerncijfers (12-uursgrens) 2003-2016. https://opend ata.cbs.nl/statline/\#/CBS/nl/dataset/71738ned/table?fromstatweb.

Statistics Netherlands. (2017). Laagopgeleide mannen vaker kinderloos. https://www.cbs.nl/nl-nl/nieuw s/2017/13/laagopgeleide-mannen-vaker-kinderloos.

Statistics Netherlands. (2019). Mijlpalen twintigers schuiven op. https://www.cbs.nl/nl-nl/nieuw s/2019/20/mijlpalen-twintigers-schuiven-op.

Steptoe, A. (2019). Happiness and health. Annual Review of Public Health, 40(4), 1-21. https://doi. org/10.1146/annurev-publhealth040218-044150.

Steverink, N. (2001). When and why frail elderly people give up independent living: The Netherlands as an example. Ageing \& Society, 21(1), 45-69. https://doi.org/10.1017/S0144686X01008066.

Steverink, N., \& Lindenberg, S. (2006). Which social needs are important for subjective wellbeing? What happens to them with aging? Psychology and Aging, 21(2), 281. https://doi. org/10.1037/0882-7974.21.2.281.

Steverink, N., Lindenberg, S., Spiegel, T., \& Nieboer, A. P. (2019). The associations of different social needs with psychological strengths and subjective well-being: An empirical investigation based on Social Production Function theory. Journal of Happiness Studies. https://doi.org/10.1007/s1090 2-019-00107-9.

Suls, J. E., Martin, R., \& Wheeler, L. (2002). Social comparison: Why, with whom, and with what effect? Current Directions in Psychological Science, 11(5), 159-163. https://doi.org/10.1111/14678721.00191 .

Suls, J. E., \& Wills, T. A. E. (1991). Social comparison: Contemporary theory and research. Hillsdale: Lawrence Erlbaum Associates.

Szell, M., \& Thurner, S. (2013). How women organize social networks different from men. Scientific Reports, 3, 1-6. https://doi.org/10.1038/srep01214.

Tay, L., \& Diener, E. (2011). Needs and subjective well-being around the world. Journal of Personality and Social Psychology, 101(2), 354-365. https://doi.org/10.1037/a0023779.

Ten Bruggencate, T. I. N. A., Luijkx, K. G., \& Sturm, J. (2017). Social needs of older people: A systematic literature review. Ageing \& Society. https://doi.org/10.1017/S0144686X17000150.

Turnbull, B., Graham, M. L., \& Taket, A. R. (2016). Social exclusion of Australian childless women in their reproductive years. Social Inclusion, 4(1), 102-115. https://doi.org/10.17645/si.v4i1.489. 
Van den Bogaard, L., Henkens, K., \& Kalmijn, M. (2014). So now what? Effects of retirement on civic engagement. Ageing \& Society, 34(7), 1170-1192. https://doi.org/10.1017/S0144686X13000019.

Van der Krieke, L., Jeronimus, B. F., Blaauw, F. J., Wanders, R. B., Emerencia, A. C., Schenk, H. M., et al. (2016). HowNutsAreTheDutch (HoeGekIsNL): A crowdsourcing study of mental symptoms and strengths. International Journal of Methods in Psychiatric Research, 25(2), 123-144. https://doi. org/10.1002/mpr.1495.

Vermunt, J. K., \& Magidson, J. (2005). Technical guide for Latent GOLD 4.0: Basic and advanced. Belmont, MA: Statistical Innovations Inc. https://research.tilburguniversity.edu/en/publications/laten t-gold-40-users-guide.

Wehmeyer, M. L., Shogren, K. A., Little, T. D., \& Lopez, S. J. (2017). Development of self- determination through the life-course. Dordrecht: Springer.

Wetzel, M., Huxhold, O., \& Tesch-Römer, C. (2016). Transition into retirement affects life satisfaction: Short-and long-term development depends on last labor market status and education. Social Indicators Research, 125(3), 991-1009. https://doi.org/10.1007/s11205-015-0862-4.

Wink, P., \& James, J. B. (2007). Annual review of gerontology and geriatrics, volume 26, 2006: The crown of life: Dynamics of the early postretirement period. New York: Springer.

Wrzus, C., Hänel, M., Wagner, J., \& Neyer, F. J. (2013). Social network changes andlLife events across the life course: A meta-analysis. Psychological Bulletin, 139(1), 53. https://doi.org/10.1037/a0028601.

Publisher's Note Springer Nature remains neutral with regard to jurisdictional claims in published maps and institutional affiliations. 